MATHEMATICS OF COMPUTATION

Volume 66, Number 217, January 1997, Pages 139-155

S 0025-5718(97)00790-4

\title{
AN A POSTERIORI ERROR ESTIMATE FOR A FIRST-KIND INTEGRAL EQUATION
}

\author{
CARSTEN CARSTENSEN
}

\begin{abstract}
In this paper we present a new a posteriori error estimate for the boundary element method applied to an integral equation of the first kind. The estimate is local and sharp for quasi-uniform meshes and so improves earlier work of ours. The mesh-dependence of the constants is analyzed and shown to be weaker than expected from our previous work. Besides the Galerkin boundary element method, the collocation method and the qualocation method are considered. A numerical example is given involving an adaptive feedback algorithm.
\end{abstract}

\section{INTRODUCTION}

The numerical treatment of an integral equation of the first kind via an adaptive scheme is studied in $[4,7,8,9,14,15,18,19,24,25]$. In this paper we sharpen results of $[4,7,8,9]$ and treat collocation and qualocation methods for the first time.

As a model example, we consider Symm's integral equation, which is equivalently related to the interior or exterior Dirichlet problem for the Laplacian in a bounded two-dimensional Lipschitz domain $\Omega$ with boundary $\partial \Omega$ of transfinite diameter $\neq 1$ and $\Gamma \subseteq \partial \Omega$ : Given $f$ find $\phi$ with

$$
-\frac{1}{\pi} \int_{\Gamma} \phi(y) \log |x-y| d s_{y}=f(x) \quad(x \in \Gamma) .
$$

A Galerkin discretization, a collocation method or a qualocation method provides us with some $\phi_{h}$ and a partition $\pi=\left\{\Gamma_{1}, \ldots, \Gamma_{N}\right\}$ of $\Gamma$ in (so-called) elements $\Gamma_{1}, \ldots, \Gamma_{N}$ with the property that the residual $R$,

$$
R(x):=f(x)+\frac{1}{\pi} \int_{\Gamma} \phi_{h}(y) \log |x-y| d s_{y} \quad(x \in \Gamma),
$$

has at least one zero in each element $\Gamma_{j}$.

The aim of this paper is to prove that this information is sufficient for an a posteriori error estimate

$$
\left\|\phi-\phi_{h}\right\|_{H^{-\alpha}(\Gamma)} \leq c(\alpha, \pi) \cdot\left(\sum_{j=1}^{N} h_{j}^{2 \alpha} \cdot\left\|\frac{\partial}{\partial s} R\right\|_{L^{2}\left(\Gamma_{j}\right)}^{2}\right)^{1 / 2}
$$

Received by the editor February 20, 1995 and, in revised form, November 6, 1995 and January 26, 1996.

1991 Mathematics Subject Classification. Primary 65N38, 65N15, 65R20, 45L10.

Key words and phrases. Integral equations, boundary element method, a posteriori error estimate, adaptive algorithm, collocation method, qualocation method.

(C) 1997 American Mathematical Society 
where prime or $\frac{\partial}{\partial s}$ denotes the derivative along $\Gamma$ with respect to the arc length. The constant $c(\alpha, \pi)$ depends on the norm (i.e., on $\alpha)$ and on the mesh $\pi$. The mesh-dependence is very weak,

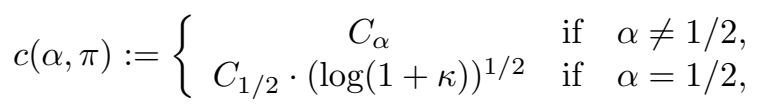

where $0 \leq \alpha \leq 1, \kappa:=\max \left\{h_{j} / h_{k}: \Gamma_{j}\right.$ is a neighbor of $\left.\Gamma_{k}\right\}$, and $C_{\alpha}$ is a universal constant depending on $\alpha$ only.

We show that this dependence of $\kappa$ for $\alpha=\frac{1}{2}$ in (1.4) is sharp. We remark that the reverse inequality of (1.3) (with a different constant $C$ ) can be proved for uniform grids as in [5].

An outline of this paper is as follows. In $\S 2$ we present an estimate as our main result in Theorem 1. We report on some applications in $\S 3$, mainly for Symm's integral equation, but point to other first-kind integral equations as well. A numerical example for the collocation method applied to Symm's integral equation from [14] is presented in $\S 4$, where we consider an adaptive mesh-refinement procedure as well. A further important application to the adaptive coupling of boundary element methods and finite element methods will appear in [6].

\section{An estimate}

We define the Sobolev space $H^{\alpha}(\Gamma)$ for an open or closed arc $\Gamma$. Let $\Omega$ be a bounded Lipschitz domain with (closed) boundary $\hat{\Gamma}=\partial \Omega$. The norm in $H^{1}(\hat{\Gamma})$ is

$$
\|v\|_{H^{1}(\hat{\Gamma})}^{2}=\|v\|_{L^{2}(\hat{\Gamma})}^{2}+\left\|v^{\prime}\right\|_{L^{2}(\hat{\Gamma})}^{2}
$$

(prime denotes derivative with respect to the arc length). Then, we define $H^{\alpha}(\hat{\Gamma})$ by (complex) interpolation of $H^{1}(\hat{\Gamma})$ and $L^{2}(\hat{\Gamma}), 0 \leq \alpha \leq 1$ (cf., e.g., [3]).

Remark 1. Equivalently, $H^{\alpha}(\hat{\Gamma})$ is the trace space

$$
H^{\alpha}(\hat{\Gamma}):=\left\{\left.v\right|_{\hat{\Gamma}}: v \in H^{\alpha+1 / 2}\left(\mathbb{R}^{2}\right)\right\} \quad(\alpha>0)
$$

of $H^{\alpha+1 / 2}\left(\mathbb{R}^{2}\right)$ defined as the standard Sobolev space [16].

For $\alpha=0$ we define $H^{0}(\hat{\Gamma})=L^{2}(\hat{\Gamma})$ and extend the scalar product in $L^{2}(\hat{\Gamma})$ to the duality pairing $\langle\cdot, \cdot\rangle$ in $H^{\alpha}(\hat{\Gamma})$ and $H^{-\alpha}(\hat{\Gamma})$ :

$$
H^{\alpha}(\hat{\Gamma}):=\left(H^{-\alpha}(\hat{\Gamma})\right)^{*} \quad(\alpha<0),
$$

with $*$ denoting the dual space.

Let $\hat{\gamma}: \mathbb{R} \rightarrow \hat{\Gamma}$ be a periodic arc length parameterization of $\hat{\Gamma}$ with length $\hat{L}$. A subarc $\omega$ of $\hat{\Gamma}$ is defined by $a<b, b-a<\hat{L}$, as the range of $\left.\hat{\gamma}\right|_{[a, b]}$. In particular, if $\Gamma$ is an open arc, we assume $\gamma:=\left.\hat{\gamma}\right|_{[0, L]}$ to be an arc length parameterization of $\Gamma$. For any subarc $\omega$,

$$
H^{\alpha}(\omega):=\left\{\left.v\right|_{\omega}: v \in H^{\alpha}(\hat{\Gamma})\right\}
$$

where the norm of $v \in H^{\alpha}(\omega)$ is defined as the minimal norm of an extension, i.e.,

$$
\|v\|_{H^{\alpha}(\omega)}:=\inf \left\{\|\hat{v}\|_{H^{\alpha}(\hat{\Gamma})}: \hat{v} \in H^{\alpha}(\hat{\Gamma}) \text { with }\left.\hat{v}\right|_{\Gamma}=v\right\} .
$$

Note that functions in $H^{1}(\omega)$ are absolutely continuous (on $\omega$ ) and their derivative (with respect to the arc length) belongs to $L^{2}(\omega)$.

A mesh $\pi=\left\{\Gamma_{1}, \ldots, \Gamma_{N}\right\}$ is defined as a partition of $\Gamma$ in elements $\Gamma_{1}, \ldots, \Gamma_{N}$ such that we have a partition of the parameter interval $0=x_{0}<x_{1}<\cdots<x_{N}=L$ 
with $\Gamma_{j}$ being the range of $\left[x_{j-1}, x_{j}\right]$ under the mapping $\gamma$. Let $h_{j}$ denote the length of $\Gamma_{j}$. If $\Gamma$ is an open arc, then we further assume that the length of the complementary arc $\hat{\Gamma} \backslash \Gamma$, that is, $\hat{L}-L$, is greater than $h_{1}+h_{N}$.

Theorem 1. Assume that $f \in H^{1}(\Gamma)$ has at least one zero in each of the elements in the mesh $\pi$. Then, for $0 \leq \alpha \leq 1$ with $c(\alpha, \pi)$ given in (1.4),

$$
\|f\|_{H^{\alpha}(\Gamma)} \leq c(\alpha, \pi)\left(\sum_{j=1}^{N} h_{j}^{2(1-\alpha)} \cdot\left\|\frac{\partial}{\partial s} f\right\|_{L^{2}\left(\Gamma_{j}\right)}^{2}\right)^{1 / 2} .
$$

The proof relies on a local interpolation as in [8] and the following lemmas.

Lemma $1([14,17,21])$. Let $f_{1}, \ldots, f_{n} \in H^{\alpha}(\hat{\Gamma}), 0 \leq \alpha \leq 1$, such that $f_{j} \cdot f_{k}=0$ on $\Gamma$ whenever $1 \leq j<k \leq n$. Then,

$$
\left\|\sum_{j=1}^{n} f_{j}\right\|_{H^{\alpha}(\hat{\Gamma})}^{2} \leq C_{1} \cdot \sum_{j=1}^{n}\left\|f_{j}\right\|_{H^{\alpha}(\hat{\Gamma})}^{2} .
$$

The constant $C_{1}$ depends on $\Gamma$ but does not depend on $f_{j}$ or on $n$.

Remark 2. The lemma is proved by von Petersdorff in [17], where $H^{\alpha}(\hat{\Gamma})$ is defined by complex interpolation (cf., e.g., [3]) with $C_{1}=1$ and for $\operatorname{supp} f_{j}$ a Lipschitz domain. The lemma is used by Stephan and Suri in [21] with $C_{1}=C$, where $H^{\alpha}(\hat{\Gamma})$ is defined by real interpolation. Faermann studied the assertion and its reverse inequality [14] and she derived some constant $C_{1}$ explicitly in case $H^{\alpha}(\hat{\Gamma})$ is regarded as a manifold (transforming the norm of $H^{\alpha}(\mathbb{R})$ in the parameter interval to the manifold). - As is well known, all the abovementioned definitions of $H^{\alpha}(\hat{\Gamma})$ define the same set of functions and equivalent norms [16]. Thus, the lemma holds in all cases, but, in general, with a different constant $C_{1}$. Here, in case of complex interpolation, $C_{1}=1$.

Remark 3. We recall the interpolation estimate: For all $f \in H^{1}(\Gamma)$ and $0 \leq \alpha \leq 1$,

$$
\|f\|_{H^{\alpha}(\Gamma)} \leq C_{2} \cdot\|f\|_{L^{2}(\Gamma)}^{1-\alpha} \cdot\|f\|_{H^{1}(\Gamma)}^{\alpha} .
$$

The constant $C_{2}$ depends certainly on the domain and on the interpolation; for complex interpolation, $C_{2}=1$.

Remark 4. An equivalent norm in $H^{\alpha}(\mathbb{R})$ is given by the Sobolev-Slobodeckij norm $|\cdot|_{\alpha}$ defined by

$$
|f|_{\alpha}^{2}:=\|f\|_{L^{2}(\mathbb{R})}^{2}+\int_{\mathbb{R}} \int_{\mathbb{R}} \frac{|f(s)-f(t)|^{2}}{|s-t|^{1+2 \alpha}} d s d t \quad\left(f \in H^{\alpha}(\mathbb{R})\right) .
$$

A central role in the proof of Theorem 1 is played by hat functions $\eta_{x, y, z} \in H^{1}(\mathbb{R})$ defined, for $x<y<z$, by

$$
\eta_{x, y, z}(t):=\left\{\begin{array}{ccc}
0 & \text { if } & t \leq x \\
\frac{t-x}{y-x} & \text { if } & x \leq t \leq y, \\
\frac{z-t}{z-y} & \text { if } & y \leq t \leq z \\
0 & \text { if } & t \geq z
\end{array} \quad(t \in \mathbb{R})\right.
$$

We essentially make use of the Sobolev-Slobodeckij norm, defined in (2.4), of the hat functions. 
Lemma 2. For $0 \leq \alpha \leq 1$ there is a constant $C_{\alpha}>0$ such that for all $x<y<z$,

$$
\left|\eta_{x, y, z}\right|_{\alpha} \leq\left\{\begin{array}{cc}
C_{\alpha} \cdot\left(|y-x|^{1-2 \alpha}+|z-y|^{1-2 \alpha}\right)^{1 / 2} & \text { if } \quad \alpha \neq 1 / 2 \\
C_{1 / 2} \cdot\left(\log \left(1+\max \left\{\frac{|z-y|}{|x-y|}, \frac{|x-y|}{|z-y|}\right\}\right)\right)^{1 / 2} & \text { if } \quad \alpha=1 / 2 .
\end{array}\right.
$$

This lemma is possibly well known; a proof is sketched at the end of this section for completeness.

Proof of Theorem 1. Since $f$ has a zero $z_{j}$ in $\Gamma_{j}$, the fundamental theorem of calculus implies, for each $z \in \Gamma_{j}$,

$$
|f(z)|=\left|\int_{z_{j}}^{z} f^{\prime}(\zeta) d s_{\zeta}\right| \leq\left\|f^{\prime}\right\|_{L^{1}\left(\Gamma_{j}\right)} \leq \sqrt{h_{j}}\left\|f^{\prime}\right\|_{L^{2}\left(\Gamma_{j}\right)},
$$

where we used Cauchy's inequality in the last step. Hence, if $y_{j}:=\gamma\left(x_{j}\right) \in \Gamma_{j-1} \cap \Gamma_{j}$ and $f_{j}:=f\left(y_{j}\right)$,

$$
\left|f_{j}\right| \leq \min \left\{\sqrt{h_{j-1}}\left\|f^{\prime}\right\|_{L^{2}\left(\Gamma_{j-1}\right)}, \sqrt{h_{j}}\left\|f^{\prime}\right\|_{L^{2}\left(\Gamma_{j}\right)}\right\} .
$$

Similarly, we infer from (2.7) that

$$
\|f\|_{L^{2}\left(\Gamma_{j}\right)} \leq h_{j}\left\|f^{\prime}\right\|_{L^{2}\left(\Gamma_{j}\right)} .
$$

First, we treat the case $\Gamma$ closed (hence equal to $\hat{\Gamma}$ ) and then deduce the second case from that. Let $y_{0}=y_{N}, y_{N+1}:=y_{1}, x_{N+1}:=L+h_{1}$, and, for $j=1, \ldots, N$ and $z \in \Gamma$, define the hat function

$$
\eta_{j}(z):=\eta_{x_{j-1}, x_{j}, x_{j+1}}\left(\gamma^{-1}(z)\right) .
$$

The norm in $H^{\alpha}(\Gamma)$ and the norm $\left|f \circ \gamma^{-1}\right|_{\alpha}$, with $|\cdot|_{\alpha}$ as in (2.4), are equivalent. Therefore, Lemma 2 leads to

$$
\left\|\eta_{j}\right\|_{H^{\alpha}(\Gamma)} \leq c(\alpha, \pi) \cdot\left(h_{j-1}^{1-2 \alpha}+h_{j}^{1-2 \alpha}\right)^{1 / 2},
$$

where $c(\alpha, \pi)$ is given in (1.4). Next we split $f$ into three parts, $f=r+g_{\text {odd }}+g_{\text {even }}$,

$$
g_{\text {even }}:=\sum_{j=1, j \text { even }}^{N} f_{j} \cdot \eta_{j} \text { and } g_{\text {odd }}:=\sum_{j=1, j \text { odd }}^{N} f_{j} \cdot \eta_{j} ;
$$

$r:=f-g_{\text {odd }}-g_{\text {even }}$. We remark that $r$ has zeros $y_{1}, \ldots, y_{N}$ and $g_{\text {odd }}$ has zeros $y_{0}, y_{2}, \ldots$, while $g_{\text {even }}$ has zeros $y_{1}, y_{3}, \ldots$. We may apply Lemma 1 to $r$ by considering $r_{j}$ defined by $r_{j}:=r$ on $\Gamma_{j}$ and $r_{j}:=0$ on $\Gamma \backslash \Gamma_{j}$. By construction, $r_{j}$ is continuous at $y_{j-1}$ and $y_{j}$ and is piecewise an absolutely continuous function. Furthermore, the derivative $r_{j}^{\prime}$ belongs to $L^{2}(\Gamma)$, so that $r_{j}$ belongs to $H^{1}(\Gamma)$. According to Lemma 1,

$$
\|r\|_{H^{\alpha}(\Gamma)}^{2} \leq \sum_{j=1}^{N}\left\|r_{j}\right\|_{H^{\alpha}(\Gamma)}^{2} \leq \sum_{j=1}^{N}\|r\|_{L^{2}\left(\Gamma_{j}\right)}^{2(1-\alpha)}\|r\|_{H^{1}\left(\Gamma_{j}\right)}^{2 \alpha},
$$

where we used (2.3) and that $r_{j}$ is zero outside $\Gamma_{j}$. Since $r$ vanishes at the endpoints of $\Gamma_{j}$, we find, as in (2.9),

$$
\|r\|_{L^{2}\left(\Gamma_{j}\right)} \leq h_{j}\left\|r^{\prime}\right\|_{L^{2}\left(\Gamma_{j}\right)} .
$$

By definition of $r$ we have $r=f-f_{j-1} \cdot \eta_{j-1}-f_{j} \cdot \eta_{j}$ on $\Gamma_{j}$. Thus, by the triangle inequality and by $\left\|\eta_{j-1}^{\prime}\right\|_{L^{2}\left(\Gamma_{j}\right)}=\left\|\eta_{j}^{\prime}\right\|_{L^{2}\left(\Gamma_{j}\right)}=h_{j}^{-1 / 2}$,

$$
\left\|r^{\prime}\right\|_{L^{2}\left(\Gamma_{j}\right)} \leq\left\|f^{\prime}\right\|_{L^{2}\left(\Gamma_{j}\right)}+h_{j}^{-1 / 2}\left|f_{j-1}\right|+h_{j}^{-1 / 2}\left|f_{j}\right| \leq 3\left\|f^{\prime}\right\|_{L^{2}\left(\Gamma_{j}\right)}
$$


because of (2.8). By (2.13)-(2.15),

$$
\|r\|_{H^{\alpha}(\Gamma)}^{2} \leq 9 \sum_{j=1}^{N} h_{j}^{2(1-\alpha)}\left(1+h_{j}^{2}\right)^{\alpha}\left\|f^{\prime}\right\|_{L^{2}\left(\Gamma_{j}\right)}^{2} .
$$

Arguing for $g_{\text {odd }}$ and $g_{\text {even }}$ as in (2.13) we conclude from Lemma 1 that

$$
\left\|g_{\text {even }}\right\|_{H^{\alpha}(\Gamma)}^{2} \leq \sum_{j=1, j \text { even }}^{N}\left|f_{j}\right|^{2} \cdot\left\|\eta_{j}\right\|_{H^{\alpha}(\Gamma)}^{2}
$$

and an analogous estimate for $g_{\text {odd }}$. Combining the two estimates and using (2.8) and (2.11), we finally derive

$$
\left\|g_{\text {even }}\right\|_{H^{\alpha}(\Gamma)}^{2}+\left\|g_{\text {odd }}\right\|_{H^{\alpha}(\Gamma)}^{2} \leq 2 c(\alpha, \pi)^{2} \sum_{j=1}^{N} h_{j}^{2-2 \alpha}\left\|f^{\prime}\right\|_{L^{2}\left(\Gamma_{j}\right)}^{2} .
$$

According to the triangle inequality, we get, for $f=r+g_{\text {odd }}+g_{\text {even }}$, from (2.16) and (2.17), that

$$
\|f\|_{H^{\alpha}(\Gamma)} \leq 6(1+c(\alpha, \pi))\left(\sum_{j=1}^{N}\left(1+h_{j}^{2}\right)^{\alpha} h_{j}^{2-2 \alpha}\left\|f^{\prime}\right\|_{L^{2}\left(\Gamma_{j}\right)}^{2}\right)^{1 / 2} .
$$

This proves the theorem in case $\Gamma$ is a closed arc.

It remains to consider the case that $\Gamma$ is an open arc. Then, the norm of $f$ is defined by extension such that we define an upper bound of $\|f\|_{H^{\alpha}(\Gamma)}$ if we extend $f$ to $\hat{f} \in H^{1}(\hat{\Gamma})$ by reflection at the endpoints of $\Gamma$, i.e.,

$$
\begin{array}{cl}
\hat{f}(\hat{\gamma}(s)):=f(\hat{\gamma}(-s)) \quad \text { if } \quad-x_{1}^{*} \leq s \leq 0, \\
\hat{f}(\hat{\gamma}(s)):=f(\hat{\gamma}(L-s)) \quad \text { if } \quad L \leq s \leq 2 L-x_{N}^{*},
\end{array}
$$

where $\gamma\left(x_{j}^{*}\right)$ is a zero of $f$ in $\Gamma_{j}$. Hence, we extend $\hat{f}$ by zero outside of $\Gamma^{*}:=$ $\hat{\gamma}\left[-x_{1}^{*}, 2 L-x_{N}^{*}\right]$. Note that $\Gamma^{*}$ is an open subarc of $\hat{\Gamma}$ because of $\hat{L}-L>h_{1}+h_{N}$. Therefore, $\hat{f}$ belongs to $H^{1}(\hat{\Gamma})$ and has zeros in each of the elements

$$
\Gamma_{0}:=\hat{\gamma}\left[-h_{1}, 0\right], \Gamma_{1}, \ldots, \Gamma_{N}, \Gamma_{N+1}:=\hat{\gamma}\left[L, L+h_{N}\right], \Gamma_{N+2}:=\hat{\Gamma} \backslash \Gamma^{*} .
$$

In other words, we have a partition of the closed arc $\hat{\Gamma}$ and a function $\hat{f}$ in $\hat{\Gamma}$. Therefore, we may apply the proven first case of the theorem and obtain an estimate like (2.1); actually it is (2.1) when we add contributions of the element $\Gamma_{0}$ (which is smaller than the contribution of $\Gamma_{1}$ ), of $\Gamma_{N+1}$ (which is smaller than the contribution of $\Gamma_{N}$ ) and of $\Gamma_{N+2}$ (which is zero). Thus, $\|f\|_{H^{\alpha}(\Gamma)} \leq\|\hat{f}\|_{H^{\alpha}(\hat{\Gamma})}$ is bounded by twice (2.1). It remains to consider the case $\alpha=1 / 2$, where a different $\kappa$ in the new mesh (2.19) on $\hat{\Gamma}$ might cause another constant. A closer look at the above arguments shows that the hat functions at the endpoints of $\Gamma^{*}$ do not appear because $\hat{f}$ vanishes there. Consequently, the norms of the related hat functions are not involved; we in fact may consider the same constant $c(\alpha, \pi)$ in the new situation (2.19). This concludes the proof of Theorem 1 in the second case.

Remark 5. We stress that (2.8) and (2.9) imply (2.1), so the condition on the zeros of $f$ is needed to ensure (2.8) and (2.9) only. Thus, Theorem 1 can be generalized, replacing the condition on the zeros of $f$ by the inequalities (2.8) and 
(2.9). Moreover, assuming weaker forms of (2.8) and (2.9), we get a (weaker) estimate of the form (2.1) following the arguments in the proof of Theorem 1.

We conclude this section with some remarks on the proof of Lemma 2.

Proof of Lemma 2. It is sufficient to consider $x=-a, y=0$, and $z=b$ with $a, b>0$, and $\eta:=\eta_{-a, 0, b}$. Since $\|\eta\|_{L^{2}(-\infty, \infty)}=(a+b) / 3$, the proof relies on a direct computation of the integral

$$
I:=\int_{-\infty}^{+\infty} \int_{-\infty}^{+\infty} \frac{|\eta(s)-\eta(t)|^{2}}{|s-t|^{1+2 \alpha}} d s d t
$$

The domain of integration $(-\infty, \infty)$ is split into four parts $(-\infty,-a),(-a, 0),(0, b)$, and $(b, \infty)$, according to the piecewise definition of $\eta$. Each of these integrals is an analytic function which can be computed by hand.

A (laborious) Maple calculation of the resulting 12 nonzero contributions for $\alpha=1 / 2$ reveals

$$
I=\left(1+\frac{b}{a}\right) \cdot \log \left(1+\frac{a}{b}\right)+\left(1+\frac{a}{b}\right) \cdot \log \left(1+\frac{b}{a}\right) \quad(\alpha=1 / 2) .
$$

Define $k:=\max \left\{\frac{a}{b}, \frac{b}{a}\right\}$. Then, (2.20) shows

$$
I \leq 2(1+1 / k) \cdot \log (1+k) \leq 4 \cdot \log (1+k),
$$

which proves the assertion in Lemma 2 for $\alpha=1 / 2$.

We report on the case $\alpha \neq 1 / 2,0<\alpha<1$, in the sequel. The abovementioned 12 integrals can be transformed into four types of integrals $I_{1}, \ldots, I_{4}$ which are estimated below, where the constants $C_{1}, \ldots, C_{4}$ depend only on $\alpha \neq 1 / 2$ but not on $a$ or $b$. The first type of integral is

$$
I_{1}:=\int_{a}^{\infty} \int_{0}^{a} \frac{|1-x / a|^{2}}{|x-y|^{1+2 \alpha}} d x d y=a^{1-2 \alpha} \int_{1}^{\infty} \int_{0}^{1} \frac{|1-x|^{2}}{|x-y|^{1+2 \alpha}} d x d y=a^{1-2 \alpha} \cdot C_{1},
$$

where $C_{1}:=\int_{1}^{\infty} \int_{0}^{1} \frac{|1-x|^{2}}{|x-y|^{1+2 \alpha}} d x d y \leq 1 /\left(2 \alpha(1-2 \alpha)^{2}\right)<\infty$. The second type, obtained, e.g., after the substitution of $x$ into $-x$, is

$$
I_{2}:=\int_{a}^{\infty} \int_{0}^{b} \frac{|1-x / b|^{2}}{|x+y|^{1+2 \alpha}} d x d y=: I_{2 a}+I_{2 b}
$$

with

$$
I_{2 a}:=\int_{b}^{\infty} \int_{0}^{b} \frac{|1-x / b|^{2}}{|x+y|^{1+2 \alpha}} d x d y=b^{1-2 \alpha} \int_{1}^{\infty} \int_{0}^{1} \frac{|1-x|^{2}}{|x+y|^{1+2 \alpha}} d x d y \leq C_{2} b^{1-2 \alpha}
$$

where $C_{2}:=\int_{1}^{\infty} \int_{0}^{1} \frac{d x d y}{|x+y|^{1+2 \alpha}} \leq 2 /(\alpha|1-2 \alpha|)<\infty$. The second contribution is, for $a<b$ only,

$$
\begin{aligned}
I_{2 b}:=\int_{a}^{b} & \int_{0}^{b} \frac{|1-x / b|^{2}}{|x+y|^{1+2 \alpha}} d x d y \leq \int_{a}^{\infty} \int_{0}^{a} \frac{|1-x / a|^{2}}{|x+y|^{1+2 \alpha}} d x d y \\
& +\int_{a}^{b} \int_{a}^{b} \frac{d x d y}{|x+y|^{1+2 \alpha}} d x d y \leq a^{1-2 \alpha} C_{2}+\frac{1}{2 \alpha|1-2 \alpha|}\left(a^{1-2 \alpha}+b^{1-2 \alpha}\right) .
\end{aligned}
$$

In the last term we used 


$$
\int_{a}^{b} \int_{a}^{b} \frac{d x d y}{|x+y|^{1+2 \alpha}} d x d y \leq\left\{\begin{array}{ccc}
\frac{b^{1-2 \alpha}}{2 \alpha(1-2 \alpha)} & \text { if } & 1>2 \alpha \\
\frac{a^{1-2 \alpha}}{\alpha(2 \alpha-1)} & \text { if } & 1<2 \alpha .
\end{array}\right.
$$

Altogether,

$$
I_{2} \leq\left(C_{2}+(\alpha|1-2 \alpha|)^{-1}\right) \cdot\left(a^{1-2 \alpha}+b^{1-2 \alpha}\right) .
$$

The third type of integral is

$$
I_{3}:=\int_{0}^{a} \int_{0}^{a} \frac{|x / a-y / a|^{2}}{|x-y|^{1+2 \alpha}} d x d y=a^{1-2 \alpha} C_{4},
$$

where $C_{4}:=\int_{0}^{1} \int_{0}^{1}|x-y|^{1-2 \alpha} d x d y \leq 2 /(1-\alpha)<\infty$.

The remaining contribution is, essentially,

$$
I_{4}:=\int_{0}^{a} \int_{0}^{b} \frac{|x / b-y / a|^{2}}{|x+y|^{1+2 \alpha}} d x d y=: I_{4 a}+I_{4 b} .
$$

We may, and will, assume without loss of generality that $a \leq b$. Then,

$$
\begin{array}{r}
I_{4 a}=\int_{0}^{a} \int_{0}^{a} \frac{|x / b-y / a|^{2}}{|x+y|^{1+2 \alpha}} d x d y=a^{1-2 \alpha} \cdot \int_{0}^{1} \int_{0}^{1} \frac{|x-y \cdot a / b|^{2}}{|x+y|^{1+2 \alpha}} d x d y \\
=a^{1-2 \alpha} \cdot \int_{0}^{1} \int_{y}^{y+1}(z-y-y \cdot a / b)^{2} \cdot z^{-1-2 \alpha} d x d y \\
\leq a^{1-2 \alpha} \cdot \int_{0}^{1} \int_{y}^{y+1} z^{1-2 \alpha} d x d y+a^{1-2 \alpha} \cdot(a / b)^{2} \int_{0}^{1} \int_{y}^{y+1} y^{2} \cdot z^{-1-2 \alpha} d x d y \\
\leq a^{1-2 \alpha} \cdot(2 /(1-\alpha)+1 /(2 \alpha))
\end{array}
$$

and

$$
I_{4 b}=\int_{a}^{b} \int_{0}^{a} \frac{|x / b-y / a|^{2}}{|x+y|^{1+2 \alpha}} d x d y \leq a^{1-2 \alpha} \int_{1}^{\infty} \int_{0}^{1} \frac{d x d y}{|x+y|^{1+2 \alpha}} \leq a^{1-2 \alpha} C_{2} .
$$

Altogether,

$$
I_{4} \leq a^{1-2 \alpha} \cdot\left(C_{2}+2 /(1-\alpha)+1 /(2 \alpha)\right) .
$$

To summarize, one can find the claimed estimate by dealing with 12 nonzero integrals which are transformed into four types $I_{1}, \ldots, I_{4}$ and estimated as above. In this way, one proves the lemma.

Remark 6. Considering a hat function $\eta_{j}$ as in the proof of Theorem 1, one verifies that the mesh-dependence in $c(\alpha, \pi)$ is sharp.

\section{Applications}

Assume $A$ is a pseudodifferential operator of order $\alpha \in \mathbb{R}$ on the closed arc $\Gamma$. Suppose there exists some $s \in \mathbb{R}$ with $0 \leq s \leq 1$ such that

$$
A: H^{s+\alpha}(\Gamma) \rightarrow H^{s}(\Gamma) \text { is linear, bounded, injective, surjective. }
$$

Hence, for $f \in H^{s}(\Gamma)$ there exists a unique solution $u \in H^{s+\alpha}(\Gamma)$ of

$$
A u=f \text {. }
$$


Assume we are given a mesh $\pi=\left\{\Gamma_{1}, \ldots, \Gamma_{N}\right\}$ on $\Gamma$ and a function $u_{h} \in H^{s+\alpha}(\Gamma)$ with the property that the residual $R:=A u_{h}-f$ belongs to $H^{1}(\Gamma)$ and that $R$ has a zero on each element $\Gamma_{j}$. Then, according to Theorem 1,

$$
\left\|u-u_{h}\right\|_{H^{s+\alpha}(\Gamma)} \leq c(s, \pi) \cdot\left\|h^{1-s} \frac{\partial}{\partial s} R\right\|_{L^{2}(\Gamma)} .
$$

Here, $h$ is considered as the piecewise constant function with $h_{\Gamma_{j}}=h_{j}$. (For a proof of (3.3) notice that $A^{-1}$ is bounded, say by $C_{A^{-1}}$, owing to the inverse operator theorem, and so $\left\|u-u_{h}\right\|_{H^{s+\alpha}(\Gamma)} \leq C_{A^{-1}}\|R\|_{H^{s}(\Gamma)}$. Thus, (3.3) follows from Theorem 1.)

In the sequel, we consider a few examples where (3.3) holds. The first model case is the single-layer potential operator $A=V, V \phi$ defined as the left-hand side in (1.1). (We consider $\phi=u$ and $\phi_{h}=u_{h}$ in this section for $A=V$ to unify notation.) The condition (3.1) is satisfied with $s=-1 / 2, \alpha=-1$ provided the capacity of $\Gamma$ is not equal to 1 (cf., e.g., [13, 22, 20, 23]; sufficient is, e.g., that $\Omega$ is included in a unit disc, and hence this is achievable by scaling of the domain). By [12], (3.1) is actually true for any $s$ in the unit interval, even for a Lipschitz boundary $\Gamma$. Moreover, if $f \in H^{1}(\Gamma)$ and $u_{h} \in L^{2}(\Gamma)$, then $R \in H^{1}(\Gamma)$. $\left(R \in H^{1 / 2+\epsilon}(\Gamma)\right.$ for some $\epsilon>0$ would be sufficient.) So it remains to study why the residual $R$ has a zero on each element.

Example 1 (Galerkin method I: discontinuous trial functions). Assume that $S_{h}$ is a finite-dimensional subspace of $L^{2}(\Gamma)$ such that, for any element $\Gamma_{j}$, we have at least one element $\eta_{j} \in S_{h}$ which satisfies

$$
\eta_{j}>0 \text { on } \Gamma_{j} \text { and } \quad \eta_{j}=0 \text { on } \Gamma \backslash \Gamma_{j} \quad(1 \leq j \leq N) .
$$

(In practical examples $S_{h}$ is some spline space such that $\eta_{j}=1$ on $\Gamma_{j}, \eta=0$ on $\Gamma \backslash \Gamma_{j}$, belongs to $S_{h}$.)

Then, the Galerkin solution $u_{h} \in L^{2}(\Gamma)$ satisfies

$$
\int_{\Gamma}\left(A u_{h}-f\right) w_{h} d s=0 \quad \text { for all } \quad w_{h} \in S_{h},
$$

and hence (3.3). (For a proof notice that, by (3.5), the integral of $R \cdot \eta_{j}$ is zero. By (3.4), the continuous function $R$ has a zero on $\Gamma_{j}$.)

Example 2 (Galerkin method II: continuous trial functions). In case of continuous trial functions, we may fail to find $\eta_{j}$ satisfying (3.4). (For example, in case $S_{h}=S_{\pi}^{1}(\Gamma)$, the continuous piecewise linear splines, the hat functions are supported on two neighboring elements.) Therefore, we assume in this example that $S_{h}$ includes nonnegative functions $\eta_{1}, \ldots, \eta_{J}$ and that there exists a mesh $\hat{\pi}=\left\{\hat{\Gamma}_{1}, \ldots, \hat{\Gamma}_{J}\right\}$ with $\operatorname{supp} \eta_{j} \subseteq \hat{\Gamma}_{j} \subset \Gamma$ for $j=1, \ldots, J$. As in the previous example, we conclude from (3.5) that $R$ has a zero on each element in $\hat{\pi}$, and so Theorem 1 leads to

$$
\left\|u-u_{h}\right\|_{H^{s+\alpha}(\Gamma)} \leq c(s, \hat{\pi}) \cdot\left(\sum_{j=1}^{J} \hat{h}_{j}^{2(1-s)} \cdot\left\|\frac{\partial}{\partial s} R\right\|_{L^{2}\left(\hat{\Gamma}_{j}\right)}^{2}\right)^{1 / 2} .
$$

To obtain the estimate (3.3), we further suppose that at most $d$ elements of $\pi$ are needed to cover one element of $\hat{\pi}$ and that conversely each element of $\hat{\pi}$ contains at least one element of $\pi$. Then, for all $\hat{\Gamma}_{j} \in \hat{\pi}$ and $\Gamma_{k} \in \pi$ with $\Gamma_{k} \subset \hat{\Gamma}_{j}$ there holds 
$h_{k} \leq \hat{h}_{j} \leq d \cdot \kappa^{d-1} \cdot h_{k}$. This and (3.6) prove the estimate (3.3) with replacing the constant $c(s, \pi)$ by

$C_{s} \cdot d^{1-s} \cdot \kappa^{d(1-s)}$ if $s \neq 1 / 2$ and $C_{1 / 2} \cdot d^{1 / 2} \cdot \kappa^{d / 2} \cdot\left(\log \left(1+d \cdot \kappa^{d}\right)\right)^{1 / 2}$ if $s=1 / 2$.

To illustrate this example, we consider $S_{h}=S_{\pi}^{1}(\Gamma)$ and define a coarser mesh $\hat{\pi}$ by $J:=\lfloor N / 2\rfloor$ and $\hat{\Gamma}_{j}:=\overline{\Gamma_{2 j-1} \cup \Gamma_{2 j}}$ for $j=\{1, \ldots, J-1\}$ or for $j=J$ if $N$ is even, and otherwise $\hat{\Gamma}_{J}:=\overline{\bigcup_{k=J, J-1, J-2} \Gamma_{k}}$. Thus, $d=2$ if $N$ is even, and $d=3$ if $N$ is odd. Finally, we stress that similar constructions are also possible for higher-order spline functions, even with higher smoothness properties.

Example 3 (Qualocation method). As in Example 1, let $S_{h}$ satisfy (3.4). Then, the qualocation method determines $u_{h} \in L^{2}(\Gamma)$ with

$$
\sum_{j=1}^{N} \sum_{i=1}^{k_{j}} \omega_{i j} \cdot\left(A u_{h}-f\right)\left(x_{i j}\right) \cdot w_{h}\left(x_{i j}\right)=0 \quad \text { for all } \quad w_{h} \in S_{h},
$$

where $x_{i j}$ are $k_{j}$ simple nodes on $\Gamma_{j}$ and $\omega_{i j}$ are positive weights. The condition (3.8) is simply a numerical approximation of the Galerkin condition (3.5) by some quadrature rules which are properly developed for integral equations in [11]. Since the weights are positive, (3.8) implies that, for a fixed element $\Gamma_{j}$, some values of $R\left(x_{i j}\right)$ are $\geq 0$ and some are $\leq 0$. By continuity of $R, R$ has a zero on $\Gamma_{j}$, thus (3.3) holds.

In case (3.4) is violated but there exist nonnegative ansatz functions with a larger support, we can proceed as in Example 2 and obtain either estimate for a coarser mesh $\hat{\pi}$ or (3.3) with larger constants as in (3.7).

Example 4 (Collocation method). In the notation of Example 3, (3.8) is a collocation method if $k_{j}=1, \omega_{j}=1$, i.e., if the residual $R$ is zero at a given node $x_{1 j} \in \Gamma_{j}$. Hence (even if (3.4) is not satisfied), (3.3) holds.

We remark that, even in the case of midpoint collocation on polygons $\left(S_{h}\right.$ the piecewise constants and $x_{1 j}$ is the midpoint of $\Gamma_{j}$ ), an a priori error estimate in $H^{s+\alpha}(\Gamma)$ seems unknown; only a tricky convergence result [10] in mesh-dependent norms (equivalent to Sobolev norms for uniform meshes) serves as a motivation to apply a low-order collocation method.

Remark 7. The Arnold-Wendland lemma [1] is used to construct a posteriori error estimates for the collocation method in [14]. Hence, discontinuous piecewise constant splines as in Example 4 are excluded in [14].

The second model case is the hypersingular integral equation, equivalently related to the Neumann problem for the Laplacian,

$$
W u(x)=f(x) \quad(x \in \Gamma),
$$

where the hypersingular operator $W: H^{s}(\Gamma) \rightarrow H^{s-1}(\Gamma)$, defined by

$$
W v(x):=-\frac{1}{\pi} \frac{\partial}{\partial n_{x}} \int_{\Gamma} v(y) \frac{\partial}{\partial n_{y}} \log |x-y| d s_{y},
$$

is linear, bounded, symmetric and a Fredholm operator of index zero [12]. The operator $A=W$ is positive definite between $H_{0}^{1 / 2}(\Gamma)$ and $H_{0}^{-1 / 2}(\Gamma)$,

$$
H_{0}^{s}(\Gamma):=\left\{v \in H^{s}(\Gamma): \int_{\Gamma} v d s=0\right\} \equiv H^{s}(\Gamma) / \mathbb{R} .
$$


From [12], (3.1) is true for $\alpha=1$ and any $s$ with $-1 \leq s \leq 0$. Hence, with a little modification, we are in the situation (3.1) and (3.2).

Given a mesh $\pi$, let $S_{\pi}^{1}(\Gamma)$ be the space of continuous functions, with support in $\Gamma$, which are a polynomial of degree $\leq 1$ on each element.

Theorem 2. Let $\Gamma$ be an open or closed arc as in $\S 2$. If $f \in L^{2}(\Gamma)$ is $L^{2}(\Gamma)$ orthogonal to $S_{\pi}^{1}(\Gamma)$, then for $0 \leq \alpha \leq 1$ with $c(\alpha, \pi)$ given in (1.4),

$$
\|f\|_{H^{-\alpha}(\Gamma)} \leq c(\alpha, \pi) \cdot\left\|h^{\alpha} f\right\|_{L^{2}(\Gamma)} .
$$

Proof. First, assume $\Gamma$ to be a closed arc. The differentiation $\frac{\partial}{\partial s}$ is an isomorphism between $H_{0}^{\alpha}(\Gamma)$ and $H_{0}^{\alpha-1}(\Gamma)$ (see, e.g., [7]). Its inverse is the integration operator $I: H_{0}^{\alpha-1}(\Gamma) \rightarrow H_{0}^{\alpha}(\Gamma)$. Let $F:=I(f)$, i.e., $\frac{\partial}{\partial s} F=f$. Since $f$ is orthogonal to $\eta_{h} \in S_{\pi}^{1}(\Gamma)$, we have

$$
0=\int_{\Gamma} \eta_{h} \frac{\partial}{\partial s} F d s=-\int_{\Gamma} F \frac{\partial}{\partial s} \eta_{h} d s
$$

by using integration by parts. Hence, $F$ is $L^{2}(\Gamma)$-orthogonal to the set of $\left\{\frac{\partial}{\partial s} \eta_{h}\right.$ : $\left.\eta_{h} \in S_{\pi}^{1}(\Gamma)\right\}$, which is the set of piecewise constant functions with integral mean zero. Since $F \in H_{0}^{1}(\Gamma), F$ is orthogonal to all piecewise constant functions, and so has at least one zero on each element. Therefore, Theorem 1 can be applied to $F$ and proves the assertion. Finally, if $\Gamma$ is an open subarc of $\hat{\Gamma}$, as in $\S 2$, with endpoints $a$ and $b$, let $\hat{f}=f$ on $\Gamma$ and $\hat{f}=0$ on $\hat{\Gamma} \backslash \Gamma$, so $\hat{f} \in H_{0}^{0}(\hat{\Gamma})$. Set $\tilde{F}:=I(f)$ and $F(x):=\tilde{F}(x)-F(a)$, so $F$ vanishes on $\hat{\Gamma} \backslash \Gamma$ and (3.13) leads to $F$ being $L^{2}(\Gamma)$-orthogonal to the piecewise constants. Thus, $F$ has a zero on each element. Since $F^{\prime}=f$ and differentiation is a bounded operator from $H^{1-\alpha}(\Gamma)$ to $H^{\alpha}(\Gamma)$, we have

$$
\|f\|_{H^{-\alpha}(\Gamma)} \leq\|\hat{f}\|_{H^{-\alpha}(\hat{\Gamma})} \leq C \cdot\|\hat{F}\|_{H^{1-\alpha}(\hat{\Gamma})} .
$$

Then, the assertion follows from the estimate on $F$ in Theorem 1.

Remark 8. As indicated in the proof of Theorem 2, the differentiation operator can be used to shift the range of interest from $0 \leq s \leq 1$ in Theorem 1 to any real $s$ (under appropriate regularity conditions on $S_{h}, u_{h}, f, A$ etc.).

Example 5 (Galerkin method for $W$ ). Assume that $S_{h}$ is a finite-dimensional subspace of $H^{1}(\Gamma)$ including $S_{\pi}^{1}(\Gamma)$, $\Gamma$ closed for notational simplicity. Given $f \in L^{2}(\Gamma)$, the Galerkin solution $u_{h} \in H^{1}(\Gamma)$ of the hypersingular integral equation (3.9) satisfies

$$
\int_{\Gamma}\left(W u_{h}-f\right) \cdot w_{h} d s=0 \quad \text { for all } \quad w_{h} \in S_{h} .
$$

Hence, $r:=W u_{h}-f$ is $L^{2}(\Gamma)$-orthogonal to $S_{\pi}^{1}(\Gamma)$, so that Theorem 2 proves, $0 \leq t \leq 1$,

$$
\left\|u-u_{h}\right\|_{H^{t}(\Gamma)} \leq c(1-t, \pi) \cdot\left\|h^{1-t} r\right\|_{L^{2}(\Gamma)} .
$$

Remark 9. Theorems 1 and 2 can be applied to a transmission problem as analyzed in [8] (also under consideration in [7]). The application of Theorems 1 and 2 follows the lines of this section, so we omit the details.

In conclusion, Theorems 1 and 2 improve on recent work $[4,7,8,9]$ in case $\Gamma$ is a one-dimensional open or closed arc. The a posteriori estimate is sharp for uniform meshes. 
Theorem 3. Let $\Gamma$ be a closed polygon and let the right-hand side $f$ in (3.2) be smooth such that the singularities of a solution $u$ are caused by corner singularities only. Suppose $\pi$ is a quasi-uniform mesh, i.e.,

$$
\max \left\{h_{j} / h_{k}: j, k=1, \ldots, N\right\} \leq C_{1},
$$

where $C_{1}$ is a global constant. In Examples $1-4$ we have $u_{h} \in S_{\pi}^{0}(\Gamma)$ and

$$
C_{2}^{-1}\left\|h^{1-s} \frac{\partial}{\partial s} R\right\|_{L^{2}(\Gamma)} \leq\left\|u-u_{h}\right\|_{H^{s-1}(\Gamma)} \leq C_{2}\left\|h^{1-s} \frac{\partial}{\partial s} R\right\|_{L^{2}(\Gamma)} .
$$

In Example 5 we have $u_{h} \in S_{\pi}^{1}(\Gamma)$ and

$$
C_{2}^{-1}\left\|h^{1-t} r\right\|_{L^{2}(\Gamma)} \leq\left\|u-u_{h}\right\|_{H^{t}(\Gamma)} \leq C_{2}\left\|h^{1-t} r\right\|_{L^{2}(\Gamma)} .
$$

Proof. The assertion follows as in [4], because there is no difference in the trial function space between Galerkin and collocation methods, and only approximation and inverse properties of the trial function space and regularity of the solution are required.

\section{Numerical EXPERIMENTS}

A posteriori error estimates are useful tools to verify the reliability of a computation and to motivate an adaptive algorithm using local upper error bounds. In this section we consider the new estimates and focus on the midpoint-collocation of Symm's integral equation method as in Example 4, because there is no rigorous a priori error estimate in energy norms $H^{-1 / 2}(\Gamma)$ for arbitrary meshes (however, cf. [10]), and so the reliability is not guaranteed without an a posteriori error control - thus the estimate (3.3) is very important to justify the numerical scheme. Numerical examples for Galerkin methods can be found, e.g., in [7, 8, 9] including algorithms of the type under consideration below.

The following numerical example is taken from [14] where we refer to for details on the implementation; see also the appendix. Let $\Omega$ be three quarter of the disc of radius $1 / 2$ centered at the origin. So, a parameterization of $\Gamma$ is $\gamma(s)=(s, 0)$ for $0 \leq s \leq 1 / 2, \gamma(s)=\frac{1}{2}(\cos (s-1 / 2), \sin (s-1 / 2))$ for $1 / 2 \leq s \leq 1 / 2+3 \pi / 4$, $\gamma(s)=(0,1+3 \pi / 4-s)$ for $1 / 2+3 \pi / 4 \leq s \leq 1+3 \pi / 4$. The right-hand side $f$ is computed by the Dirichlet data $u_{0}$ as

$$
f(x)=u_{0}(x)-\frac{1}{\pi} \int_{\Gamma} u_{0}(y) \frac{\partial}{\partial n_{y}} \log |x-y| d s_{y} \quad(x \in \Gamma) .
$$

We use $u_{0}(r, \varphi)=r^{2 / 3} \cdot \sin (2 \varphi / 3)$ as Dirichlet data in polar coordinates. The solution of the Dirichlet problem is $u=u_{0}$ (since $u_{0}$ is harmonic) and $\phi$ in (1.1) is the normal derivative of $u$. Note that the singularity of $u_{0}$ at the corner is generic for the domain $\Omega$.

In the discretization, the integral $I_{j}(x):=-\frac{1}{\pi} \int_{\Gamma_{j}} \phi(y) \log |x-y| d s_{y}$ is approximated by $I(x, a, b)$, where $\Gamma_{j}$ is replaced by the straight line segment $[a, b]$ between the end points $a$ and $b$ of $\Gamma_{j}$ (taking the chord instead of the arc). We refer to [14] for details; the numbers below are computed with functions $f$ and $I(x, a, b)$ as shown in the appendix. 
With $x_{j}$ denoting the midpoint of $\Gamma_{j}$ (on the arc), the collocation equations read, for the unknown coefficients $u_{j}$,

$$
\sum_{j=1}^{N} I_{j}\left(x_{k}\right) \cdot u_{j}=f\left(x_{k}\right) \quad(k=1, \ldots, N) .
$$

The implementation is performed in Maple, the (small) linear system is solved by Gaussian elimination.

Once $\left(u_{1}, \ldots, u_{N}\right)$ are known, the residual can be computed by $R(x):=f(x)-$ $\sum_{j=1}^{N} I_{j}(x) \cdot u_{j}$. We evaluated $R$ on each interval and computed a spline approximation of $R$ to reduce computational costs.

The discrete solution $u_{h}$ (piecewise constant with $u_{j}:=\left.u_{h}\right|_{\Gamma_{j}}$ ) for $N=10$ and the exact solution are plotted in Fig. 1a as functions on the parameter interval. The coarsest mesh is indicated there by the jumps of $u_{h}$ ( $u_{h}$ being piecewise constant). In a different scaling, the related residual $R$ is shown in Fig. $1 \mathrm{~b}$.

By using a spline approximation of $R$, the quantities

$$
a_{j}:=\left\|R^{\prime}\right\|_{L^{2}\left(\Gamma_{j}\right)} \quad(j=1, \ldots, N)
$$

can easily be computed as the upper bound $b_{N}$,

$$
b_{N}:=\left(\sum_{j=1}^{N} h_{j} \cdot a_{j}^{2}\right)^{1 / 2} .
$$

Since the solution is known, we can compute the energy error

$$
e_{N}:=\left(\sum_{j=1}^{N} \int_{\Gamma} R \cdot\left(u-u_{h}\right)\right)^{1 / 2}=\int_{\Gamma}\left(V\left(u-u_{h}\right)\right)\left(u-u_{h}\right) d s,
$$

which is equivalent to the error in the $H^{-1 / 2}(\Gamma)$-norm. Starting with the coarse mesh for $N=10$, indicated in Fig. 1a by the jumps of $u_{h}$, we define a quasiuniform discretization by successively halving of all elements giving meshes with $N=10,20,40,80, \ldots$ elements. The corresponding quantities are shown in Table 1.

TABLE 1. Numerical results for quasi-uniform meshes

\begin{tabular}{rcccc}
\hline$N$ & $e_{N}$ & $\alpha_{N}$ & $b_{N}$ & $e_{N} / b_{N}$ \\
\hline 10 & .083212 & & .16861 & .4935 \\
20 & .050715 & .714 & .09877 & .5135 \\
40 & .031698 & .677 & .06063 & .5227 \\
80 & .019925 & .669 & .03787 & .5260 \\
160 & .012544 & .667 & .02377 & .5275 \\
\hline
\end{tabular}

We observe that the error $e_{N}$ is decreasing and so the scheme seems to converge. The experimental convergence rate $\alpha_{N}$ is computed as

$$
\alpha_{N}=\frac{\log \left(e_{N^{\prime}} / e_{N}\right)}{\log \left(N / N^{\prime}\right)}
$$

where $N^{\prime}$ and $e_{N^{\prime}}$ are the corresponding values of the previous row. From Table 1 we see that a uniform mesh yields a convergence rate $2 / 3$ which, as is well known, 

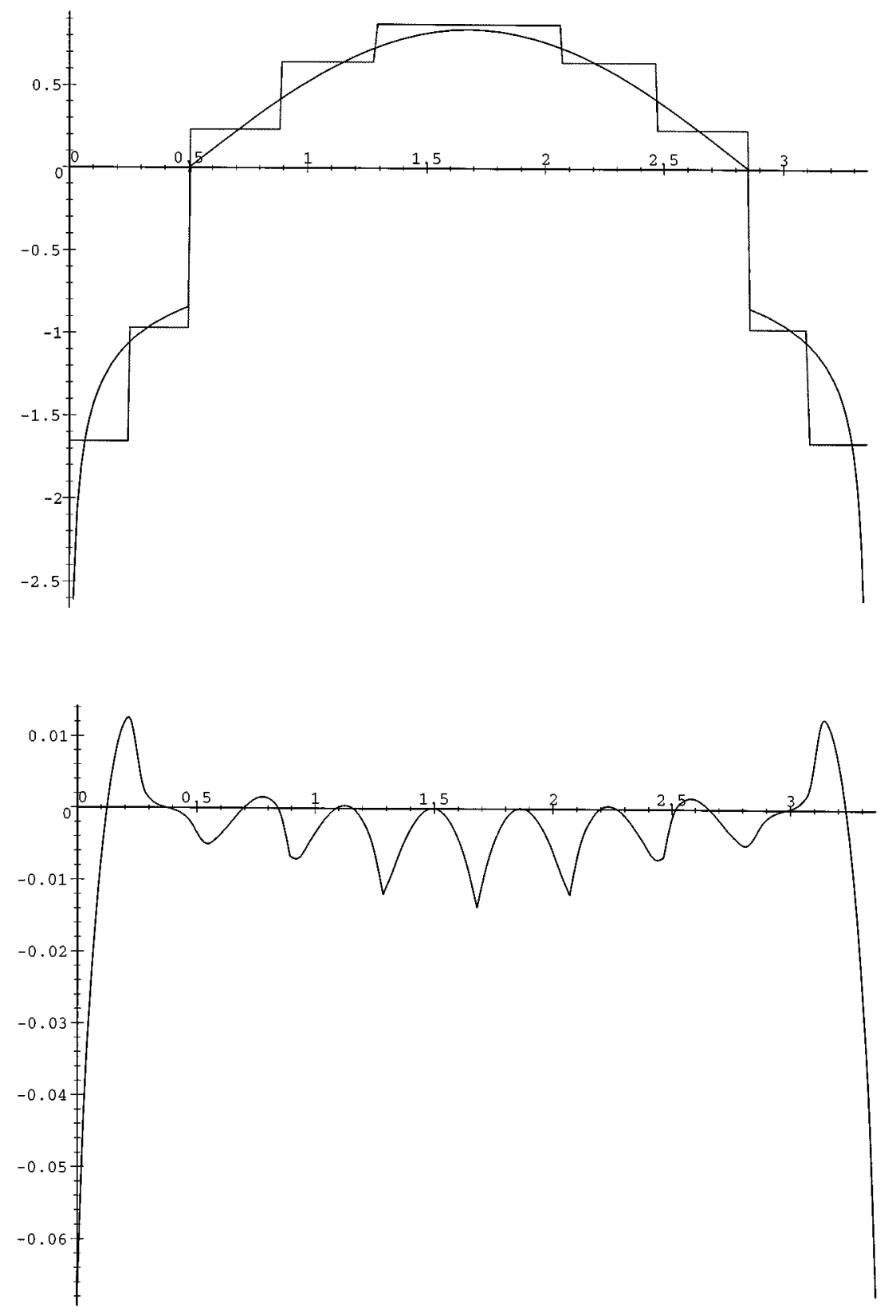

Figure 1. Exact and discrete solution in 1a (top) and residual $R$ in 1b (bottom) for $N=10$

is expected for a Galerkin scheme (see, e.g., [21, pp. $805 \mathrm{f}]$ for a discussion on the convergence rates in this example). In practical situations, we do not know $e_{N}$ but may compute $b_{N}$. Since, by Theorem 1 applied in Example 4, we have an estimate

$$
e_{N} \leq C(\log (1+\kappa))^{1 / 2} \cdot b_{N}
$$

$\left(\kappa\right.$ as in (1.4)), the values $b_{N}$ in Table 1 indicate convergence as well and justify that $u_{h}$ may be regarded as a reasonable approximation to $u$. 


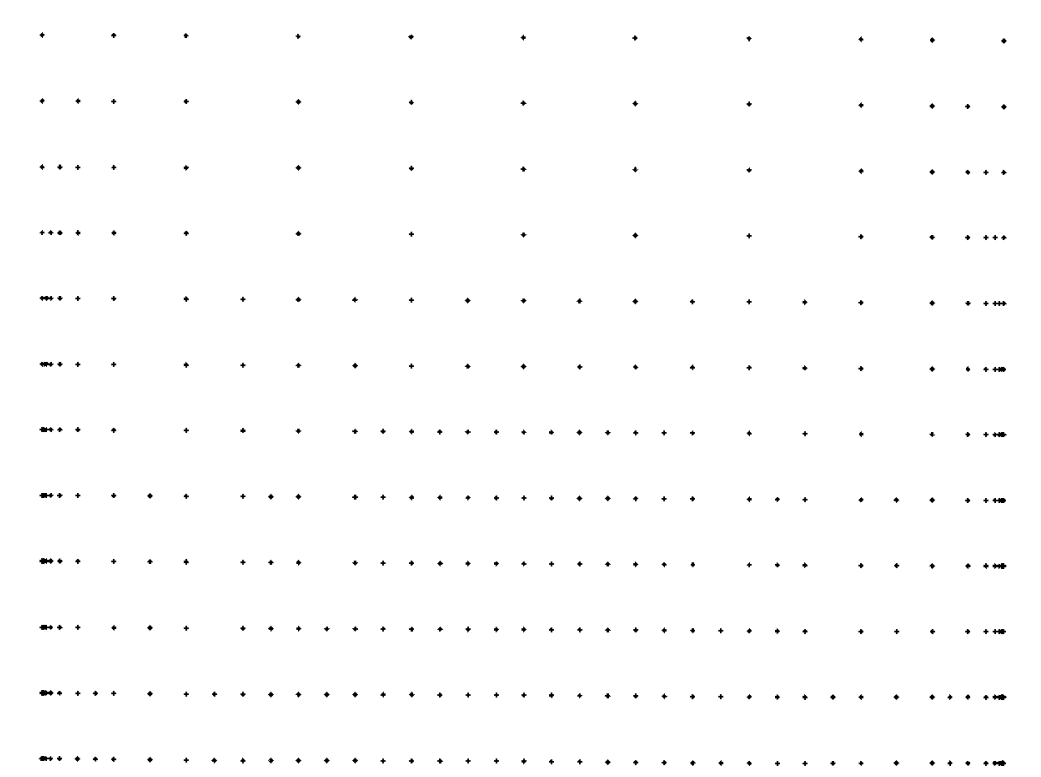

Figure 2. Meshes generated by Algorithm (A) indicated by nodes in the parameter interval

The question of efficiency is related to the quantities $e_{N} / b_{N}$ in Table 1 , which are proved to be bounded from above. The numbers in Table 1 are bounded below as well, which indicates efficiency of the estimate as proved in Theorem 3.

The final application is to adaptive algorithms. As in $[7,8,9]$ we use the size of $h_{j} a_{j}^{2}$, the contribution of $\Gamma_{j}$ to the upper bound, to decide if we should refine $\Gamma_{j}$ or not.

Algorithm (A) . Given a coarse mesh, refine it successively by halving some of its elements as follows: For any mesh $\pi$ compute $a_{1}, \ldots, a_{N}$ as defined above and refine $\Gamma_{j}$ if and only if

$$
h_{j} \cdot a_{j}^{2} \geq \theta \cdot \max _{k=1, \ldots, N} h_{k} \cdot a_{k}^{2}
$$

Remark 10. We ignored the mesh-dependence in $c(1 / 2, \pi)$ in this algorithm for simplicity. Since the dependence on $\kappa$ is very weak, this is reasonable if we deal with a few refinement steps only.

With $\theta=1 / 2$ and the coarse mesh with $N=10$ as above, Algorithm (A) determines a sequence of meshes as shown in Fig. 2, where the coarse mesh (chosen as in Fig. 1) is indicated on top by displaying the nodes in the parameter interval only. Each subsequent line represents the mesh after another step of Algorithm (A) and corresponds to the rows in Table 2 .

In the first four steps, we observe just a refinement towards the endpoints, which is reasonable in view of the singularity there. The fifth and seventh mesh show some overall refinement followed by a further local refinement towards the endpoints. For these, quite arbitrary meshes, no convergence result in the energy norm seems to be available, so we can only rely on the computable upper bound $b_{N}$ shown in Table 2 as well as other related quantities explained above. 
TABle 2. Numerical results for adapted meshes generated by Algorithm (A)

\begin{tabular}{ccccc}
\hline$N$ & $e_{N}$ & $\alpha_{N}$ & $b_{N}$ & $e_{N} / b_{N}$ \\
\hline 10 & .083212 & & .16861 & .49 \\
12 & .056021 & 2.17 & .11900 & .47 \\
14 & .040796 & 2.06 & .09164 & .44 \\
16 & .032842 & 1.62 & .07806 & .42 \\
24 & .016187 & 1.74 & .03646 & .44 \\
26 & .012974 & 2.76 & .03137 & .41 \\
34 & .010497 & 0.79 & .02272 & .46 \\
40 & .008647 & 1.19 & .01910 & .45 \\
42 & .008561 & 0.20 & .01821 & .47 \\
46 & .007017 & 2.19 & .01656 & .42 \\
52 & .004830 & 3.04 & .01269 & .38 \\
\hline
\end{tabular}

From Table 2 we infer convergence because $b_{N}$ seem to decrease to zero. Moreover, the convergence rate as well as the accuracy is remarkably improved. Efficiency is observed because $e_{N} / b_{N}$ seems to be bounded below - though there is no proof for that. In conclusion, Algorithm (A) appears to be a proper tool in this example, even for the collocation method as illustrated as follows: The initial error $e_{10}$ is reduced to $10 \%$ using 10 meshes with degrees of freedom $10,12, \ldots, 46$. The computer costs for each (small) system with $N$ elements are essentially $C \cdot N^{2}$ (postprocessing and computation of the stiffness matrix rather than the LU decomposition dominate the effort). So we have to compare $10^{2}+12^{2}+\cdots+46^{2}=8,584$ with $160^{2}=25,600$ for the finest uniform mesh which produced far worse results.

\section{APPENDIX}

This appendix briefly documents the implementation of the midpoint collocation considered in the example of $\S 4$. We refer to [14] for more details and proofs. Calculation of $f(x)$ :

$$
\begin{aligned}
& f(x)=-\frac{1}{2}|x|^{2 / 3} \sin (2 \varphi / 3)+\phi_{D}(x), \\
& \phi_{D}(x)=-\frac{3}{\pi} 2^{-8 / 3} \text { if } \quad|x|=1 / 2, \\
& \phi_{D}(x)=-\frac{3}{\pi} 2^{-8 / 3}\left(1+\frac{1}{2}\left(1-t^{2}\right) \cdot \int_{0}^{\pi} \frac{\sin \omega}{1+t^{2}-2 t \cos (3 \omega / 2)} d \omega\right) \\
& \text { if } t:=2 \cdot|x|<1 .
\end{aligned}
$$

Calculation of $I:=I(x, a, b): \alpha:=|b-a|^{2}, \beta:=-2(x-a)^{T} \cdot(b-a), \gamma:=|x-a|^{2}$, $\delta:=4 \alpha \beta-\beta^{2}, \eta:=-\frac{\beta}{2 \alpha}$;

if $\delta=0$ then

$$
\begin{aligned}
& \text { if } \eta=0 \text { or } \eta=1 \\
& \qquad \begin{aligned}
\text { then } I:=-\frac{\sqrt{\alpha}}{2 \pi}\left(\frac{1}{2} \log \alpha-1\right) \\
\quad \text { else } I:=-\frac{\sqrt{\alpha}}{2 \pi}\left(\frac{1}{2} \log (\alpha+\beta+\gamma)-1-\eta \log \left|\frac{1-\eta}{\eta}\right|\right) \mathrm{fi}
\end{aligned}
\end{aligned}
$$


else

$$
\begin{aligned}
I:=-\frac{\sqrt{\alpha}}{2 \pi}\left(\frac{1-\eta}{2} \log (\alpha\right. & +\beta+\gamma)+\frac{\eta}{2} \log (\gamma)-1 \\
& \left.+\frac{2}{\sqrt{\delta}} \cdot(\gamma+\beta \eta / 2)\left[\arctan \left(\frac{2 \alpha+\beta}{\sqrt{\delta}}\right)-\arctan \left(\frac{\beta}{\sqrt{\delta}}\right)\right]\right) \text { fi. }
\end{aligned}
$$

\section{ACKNOWLEDGEMENT}

The author thanks one referee for suggesting Example 2.

\section{REFERENCES}

1. D.N. Arnold, W.L. Wendland: On the asymptotic convergence of collocation methods. Math. Comp. 41 (1983) 349-381. MR 85h:65254

2. M. Asadzadeh, K. Eriksson: An adaptive finite element method for a potential problem. SIAM J. Numer. Anal. 31 (1994) 831-855. MR 95b:65155

3. J. Bergh, J. Löfström: Interpolation spaces. Springer Berlin 1976. MR 58:2349

4. C. Carstensen: Adaptive boundary element methods and adaptive finite element and boundary element coupling. In Proceedings Boundary Value Problems and Integral Equations on NonSmooth Domains, eds. M. Costabel, M. Dauge, S. Nicaise. Lecture notes in pure and applied mathematics 167, Marcel Dekker New York 1995, 47-58. CMP 95:03

5. C. Carstensen: Efficiency of a posteriori BEM error estimates for first-kind integral equations on quasi-uniform meshes. Math. Comp. 65 (1996), 69-84. CMP 96:03

6. C. Carstensen: A posteriori error estimate for the symmetric coupling of finite elements and boundary elements, Computing (in press), 1996.

7. C. Carstensen, E.P. Stephan: A posteriori error estimates for boundary element methods. Math. Comp. 64 (1995) 483-500. MR 95f:65211

8. C. Carstensen, E.P. Stephan: Adaptive boundary element methods for some first-kind integral equations. SIAM J. Numer. Anal. (1996), to appear.

9. C. Carstensen, E.P. Stephan: Adaptive boundary element methods for transmission problems. J. Austr. Math. Soc. Ser. B (1996), to appear.

10. G.A. Chandler: Discrete norms for the convergence of boundary element methods. Proceedings of the Center for Mathematics and its Applications. Australian National University 26 (1991) 62-78. MR 92k:65175

11. G.A. Chandler, I.H. Sloan: Spline qualocation methods for boundary integral equations. $N u-$ mer. Math. 58 (1990) 537-567. MR 91m:65323

12. M. Costabel: Boundary integral operators on Lipschitz domains: Elementary results. SIAM J. Math. Anal. 19 (1988) 613-626. MR 89h:35090

13. M. Costabel, E.P. Stephan: Boundary integral equations for mixed boundary value problems in polygonal domains and Galerkin approximation. Banach Center Publ. 15 (1985) 175-251. MR 88f:35037

14. B. Faermann: Lokale a-posteriori-Fehlerschätzer bei der Diskretisierung von Randintegralgleichungen. PhD-thesis, University of Kiel, FRG (1993).

15. N. Heuer: $h p$-Versionen der Randelementemethode. PhD-thesis, University of Hannover, FRG (1992).

16. J.L Lions, E. Magenes: Non-homogeneous boundary value problems and applications, Vol. I. Berlin-Heidelberg-New York: Springer 1972. MR 50:2670

17. T. von Petersdorff: Randwertprobleme der Elastizitätstheorie für Polyeder - Singularitäten und Approximation mit Randelementmethoden. PhD-thesis, TH Darmstadt, FRG (1989).

18. E. Rank: Adaptive boundary element methods. in: C.A. Brebbia, W.L. Wendland and G. Kuhn, eds., Boundary Elements 9, Vol. 1, 259-273. Springer Verlag Heidelberg 1987. CMP 21:03

19. J. Saranen and W.L. Wendland: Local residual-type error estimates for adaptive boundary element methods on closed curves. Appl. Anal. 48 (1993) 37-50. MR 95e:65112

20. I.H. Sloan, A. Spence: The Galerkin method for integral equations of the first kind with logarithmic kernel: Theory. IMA J. Numer. Anal. 8 (1988) 105-122. MR 90d:65230a 
21. E.P. Stephan, M. Suri: The hp-version of the boundary element method on polygonal domains with quasiuniform meshes. Math. Model. Numer. Anal. 25 (1991) 783-807. MR 92m:65154

22. E.P. Stephan, W.L. Wendland: Remarks on Galerkin and least squares methods with finite elements for general elliptic problems. Manuscripta Geodaetica 1 (1976) 93-123.

23. E.P. Stephan, W.L. Wendland, G.C. Hsiao: On the integral equation method for the plane mixed boundary value problem of the Laplacian. Math. Meth. Appl. Sci. 1 (1979) 265-321. MR 82e:31003

24. W.L. Wendland, De-hao Yu: Adaptive boundary element methods for strongly elliptic integral equations. Numer. Math. 53 (1988) 539-558. MR 89h:65194

25. W.L. Wendland and De-hao Yu: A posteriori local error estimates of boundary element methods with some pseudo-differential equations on closed curves. J. Comput. Math. 10 (1992) 273-289. MR 93d:65105

Mathematisches Seminar, Christian-Albrechts-Universität Zu Kiel, Ludewig-MeynStr. 4, D-24098 Kiel, Germany

E-mail address: cc@numerik.uni-kiel.de 